\title{
Nerves Transfer Procedure in Patients with Left Upper Extremities Weakness Following Gunshot Wounds: A Case Report
}

\author{
Indra Rukmana Tri Pratistha*, Nyoman Gede Bimantara, I. Gede Mahardika Putra, Made Bramantya Karna, \\ Anak Agung Gde Yuda Asmara, Putu Feryawan Meregawa
}

Department of Orthopaedics and Traumatology, Faculty of Medicine, Udayana University, Sanglah General Hospital, Denpasar, Bali, Indonesia Edited by: Igor Spirosk
Citation: Pratistha IRT, Bimantara NG, Putra IGM Karna MB, Asmara AAGY, Meregawa PF. Nerves transfer procedure in patients with left upper extremities weaknes lowing gunshot wounds: A case report. Open Access (1) Keywords: Gunshot wounds; Brachial plexus trauma;

Nerve transfer procedure
*Correspondence: Indra Rukmana Tri Pratistha, Department of Orthopaedics and Traumatology, Faculty Medicine, Udayana University, Sanglah Genera Indonesia. E-mail: dr_indra_pratistha@yahoo.co.id Received: 25-Jul-202 Revised: 18-Aug-2021 Accepted: 26-Aug-2021
Copyright: $\odot 2021$ Indra Rukmana Tri Pratistha Nyantya Karna, Anak Agung Gde Yuda Asmara . Autu Feryawan Meregawa did not receive any financia

Funding: This research did Competing Interest: The authors have declared that no competing interest exists Open Access: This is an open-access article distributed under the terms of the Creative Commons Attribution-

\section{Abstract}

BACKGROUND: Gunshot wounds (GSWs) to the extremities can result in damage to the neurovascular structure which results in high morbidity and loss of function. According to the Centers for Disease Control report, the incidence of non-fatal GSWs has increased in the past decade. Trauma to the brachial plexus is a type of peripheral nerve trauma that is most difficult to treat due to its complex surgical procedures. Early exploration and reconstruction of peripheral nerve trauma are still being debated to this day. However, most recommend surgical exploration when the suspicion of neurovascular trauma is very high based on clinical findings. Nerve transfer is one of the recommended methods of nerve reconstruction even in pre-ganglionic lesions. We report a case of a patient with weakness of the upper limb after a gunshot wound to his left shoulder. Based on clinical considerations and investigations, nerve transfer procedure is carried out to restore patient's shoulder function.

CASE REPORT: Male, 32 years old, working as a policeman, complained difficulty on moving his shoulder for 3 months. Patients had a history of GSWs to the left shoulder which also results in a left clavicular fracture. First aid, debridement, and fracture management were performed at Bhayangkara Hospital, Palu. Physical examination revealed winging scapula positive on his left shoulder, shoulder abduction 5/1, and hypoesthesia at left C5 level. Electromyographic examination revealed lesions on the left posterior chord and left brachial plexus. Based on clinical findings and supporting examination, we performed nerve transfers procedure from the accessory nerve to suprascapular notch. In the previous study, $63 \%$ of cases GSWs associated with nerve dysfunction. About $75 \%$ of patients with nerve palsy are associated with nerve lacerations during surgical exploration. However, many surgeons continue to recommend early exploration after GSWs to the upper extremities, especially in patients who will undergo surgical treatment for other indications. Based on this, we suggest the probable cause of brachial plexus lesions in this case resulted from gunshot wound which injures the brachial plexus or as a complication from previous procedures. Surgery that is too early can interfere with the spontaneous reinnervation process, but late surgical procedures can result in failure of reinnervation. In general, optimal time is set between 3 and 6 months after trauma. Nerve transfer is one method of reconstructing peripheral nerve lesions that can be applied to pre-ganglionic or post-ganglionic lesions.

CONCLUSION: This procedure has several benefits, namely, the proximity of the donor and the recipient nerve anatomy, shorter operating time and does not require grafts. Brachial plexus trauma due to trauma or non-trauma together has an impact on the patient's quality of life. However, advances in surgical techniques and furthe understanding of nerve physiology have led clinicians and patients to better outcomes. The current trend of treatment strategies for brachial plexus trauma is surgical reconstruction with the nerve transfer procedure.

\section{Introduction}

GSWs to the upper extremities can result in permanent nerve damage which results in morbidity and loss of function. The precise mechanism of nerve injury is multifactorial and thought to be caused by a combination of direct and indirect (heat, shock wave) trauma, making the selection of treatment often difficult. A study reported 23,152 patients with GSWs to the upper extremity between 2007 and 2012, approximately 6987 lesions accompanied by neurovascular lesion [1]. The Centers for Disease Control also reported an increase in the number of non-fatal GSWs from 23.2 per 100,000 in 2007 became 26.65 per 100,000 in 2013 and became 41.11 per 100,000 in 2017 [2]. Based on this, it is clear that the incidence of neurovascular lesions due to GSWs has also increased. Although lacerations of nerve fibers must be corrected surgically to increase the chances of recovery, it is still unclear whether the therapy is able to optimally restore the continuity of nerve fibers. In addition, even though nerve fibers have been connected, there is still a possibility of dysfunction due to glial scar and neuroma formation after the first lesion, which further complicates the determination of therapy. Nonetheless, surgeons continue to recommend exploration of GSWs, especially when suspicion of neurovascular trauma is found in clinical patients [1].

We report a case of upper extremity weakness following a gunshot wound that was referred to Sanglah General Hospital. Based on clinical, radiological examination, and ENMG, the patient was diagnosed with 
lesions on the left posterior cord and the left brachial plexus suprascapular nerve, and nerve transfer procedures were performed by the Orthopedic and Traumatology Surgery Team at Sanglah General Hospital.

\section{Case Report}

Male, 32 years old, presents with difficulty in moving his left shoulder. Complaint was felt after the patient received a gunshot wound on the left shoulder about 3 months ago. The patient also complained of numbness in his left shoulder. History of loss of consciousness, nausea, and vomiting is denied. History of hypertension, diabetes mellitus, kidney disease, and heart disease is denied.

The patient is a policeman who's looking for a victim in the middle of a forest in Palu, suddenly attacked by a mob of terrorists and got shot on his left shoulder and right lower leg. At the time of the incident, history of loss of consciousness, nausea, and vomiting was denied. The patient was taken to Bhayangkara Hospital in Palu and diagnosed with an open fracture of the left clavicle and open fracture right tibia with compartment syndrome. The patient was then treated for surgical fasciotomy, debridement, and open reduction and internal fixation-percutaneous surgery on the left clavicle and external fixation on one-third proximal of the right tibia. The patient had undergone implant revisions with bone graft due to implant failure in the middle third of the left clavicle in February (1 month before). The patient was then referred to Sanglah Hospital in March due to nerve palsy of the upper left extremity.

From physical examination, hemodynamic was stable and other examination findings were unremarkable. From neurological status, the patient was alert and meningeal signs were negative. Examination of motor function in the upper extremities results in positive winging of the scapula (Figure 1) on the left shoulder and shoulder abduction $5 / 1$. The patient was able to extend his elbows, wrists, and five fingers. Sensory function examination was found to be hypoesthesia from the left shoulder to the thumb and index finger. Sensation on the middle finger is normal. The local status of the

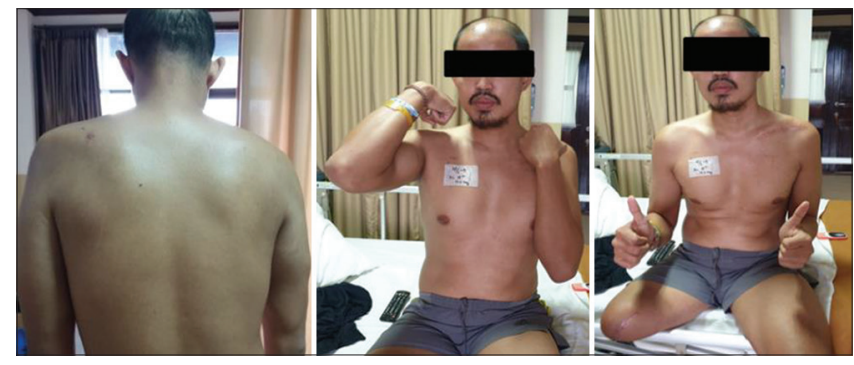

Figure 1: Suspicion of C5/C6 fiber lesions characterized by winging positive scapula on the left shoulder, shoulder abduction 5/1, and thumb extension $5 / 5$ left shoulder is obtained by inspection, the scapula is prominent compared to the right side, palpation is within normal limits, and evaluation of ranges of motion is limited both active and passive. Clinical findings were subsequently confirmed by electromyography (EMG), and the conclusions of the left posterior cord and left brachial plexus nerve lesions were concluded.

Chest and left shoulder X-ray was taken as soon as patient received a gunshot wound at the Bhayangkara Hospital in Palu about 3 months ago. From these photos, it is clear that complete fracture in one-third of the middle of the left (Figure 2). All costae is good, heart and lung showed normal impression.

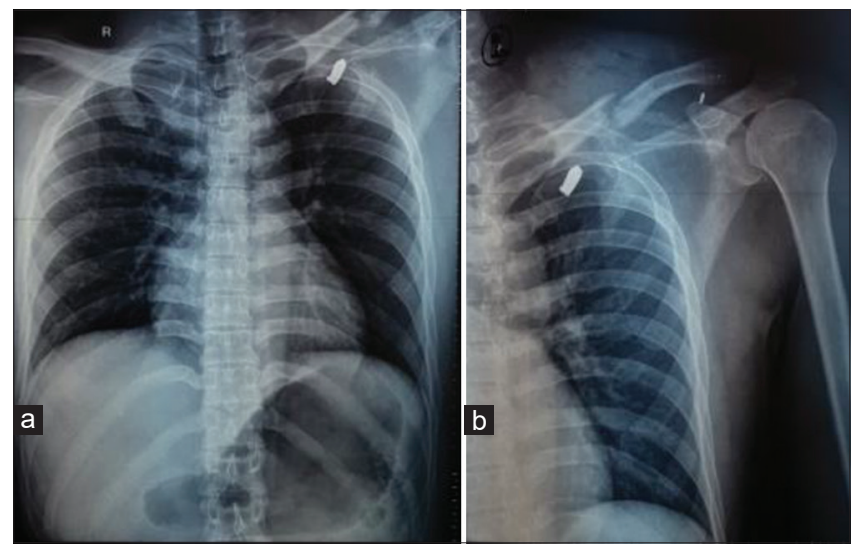

Figure 2: (a) Chest X-ray anteroposterior $(A P)$ and (b) left shoulder $X$-ray AP showing a fracture of the middle third left clavicle. Cor pulmo with a normal impression

The procedure is performed with patient in pronated position. Before disinfection, measurements of the accessory nerve (N.XI) and suprascapular notch were done. The incision is carried out in a posterior approach and followed by an accessory nerve exploration using the nerve stimulator and suprascapular notch exploration (Figure 3). The next action is cutting and the nerve transfers the accessory nerve to the suprascapular notch (Figure 3).
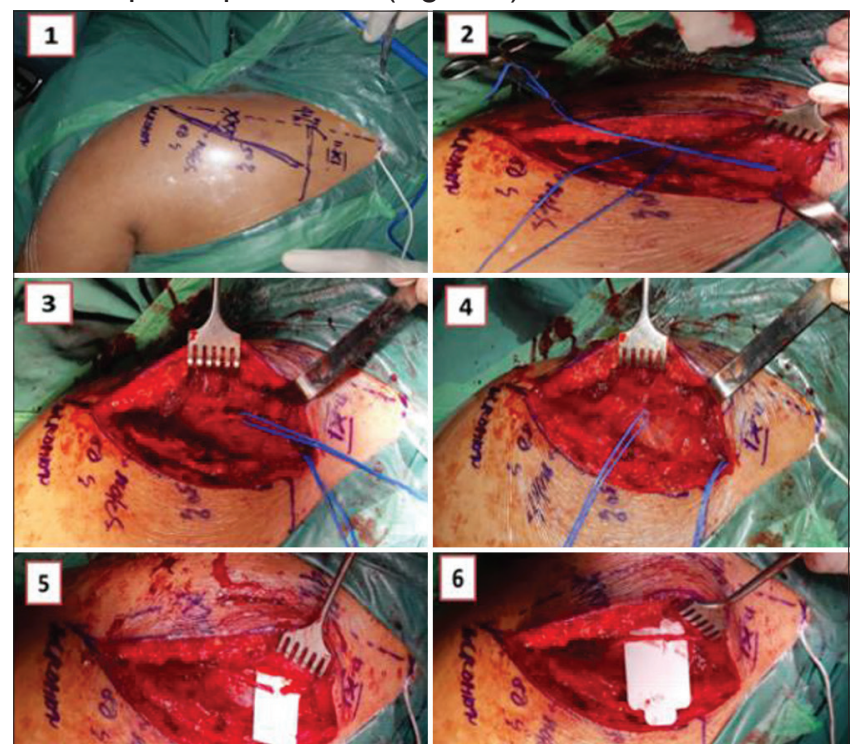

Figure 3: Surgical procedure with a posterior approach, cutting and nerve transfer procedure of the accessory nerve, and suprascapular notch 


\section{Discussion}

GSWs are often associated with orthopedic complications such as fractures, compartment syndrome, infection, nerve palsy, soft-tissue compromise, and lead toxicity [3]. Nerve damage after a gunshot wound can occur due to direct trauma from the bullet itself, or indirectly due to thermal injury, secondary laceration due to a fracture fragment, or due to compression from swelling or due to subacute scar formation. In addition, shock wave, cavitation, and bullet pathways when penetrating tissue contribute to damage to surrounding soft tissue, including nearby nerve structures. Panell et al. study also showed that the presence of a fracture after a gunshot wound to the upper extremity would increase the risk of nerve lesions by 2.6 times $(p<0.05)[1]$. As found in this case, the patient had gunshot wound to the left shoulder and result in patient having an open fracture of the left ocular clavicle with suspicion leading to the presence of lesions on the left brachial plexus.

Brachial plexus trauma is a type of peripheral nerve trauma that is most difficult to treat due to its complex surgical procedures. GSWs are the second most common cause of brachial plexus trauma [4]. The brachial plexus runs within the interscalene triangle bounded by the anterior scalene muscle anteriorly, the middle scalene muscle posteriorly, and the superior border of the first rib inferiorly. It traverses the posterior triangle of the neck, formed by the clavicle inferiorly, the trapezius muscle laterally, and the posterior border of sternocleidomastoid muscle medially. Then, it passes laterally over the first rib and enters the axilla to supply the upper limb.

Root injury is defined as avulsion of the spinal cord in the pre-ganglionic root or dorsal ganglion area of the vertebral foramen. Brachial plexus trauma can be divided into pre-ganglionic, post-ganglionic, and combination of both lesions. Pre-ganglionic injury involves avulsion of nerve roots, a structure that connects peripheral nerves to the central nervous system. So that the affected nerve fibers cannot be used as nerve sources for reconstruction, so an alternative method is needed. Whereas lesions located in the distal ganglion are called post-ganglionic injury, which is further differentiated into supra- and infrascapularis lesions. Suprascapular lesions consist of lesions of the spinal cord, nerve trunks, or nerve divisions. Whereas the infrascapular lesions consist of chordae or terminal branches lesions. Based on this, the connection of nerve fibers in post-ganglionic injury with the central nervous system is still intact and the nerve can still be used as a source of axons. Upper extremity position in the patients is the most important point for understanding the mechanism of injury to the brachial plexus, especially when trauma occurrs [5].
Level of lesion can be suspected by clinical manifestations, such as active and passive movements of the shoulder, upper arm, forearm, hand, and wrist. Muscle grading systems such as Louisiana State University Health Science Center and Medical Research Council grade system have been widely used to evaluate the power of muscles. Upper brachial plexus lesions $(\mathrm{C} 5, \mathrm{C} 6)$ cause paralysis of the shoulder muscles and biceps. Paralysis of the shoulder muscles is accompanied by an inability to arm abduction or weakness of the rhomboid muscle showing lesions at the $\mathrm{C} 5$ level. If elbow flexion and wrist extension are disturbed or there is a winging scapula, C6 involvement must be considered. Furthermore, there will be functional loss in finger and thumb flexion if the $\mathrm{C} 7$ is involved. The findings of neurological and functional abnormalities in brachial plexus lesions are summarized in Table 1 [5].

Table 1: Neurological findings on brachial plexus lesion

\begin{tabular}{|c|c|c|c|}
\hline & Muscle affected & Functional loss & Sensory loss \\
\hline C5/C6 & $\begin{array}{l}\text { Deltoid, supra/infraspinatus, } \\
\text { biceps, brachialis, } \\
\text { coracobrachialis, (+/-) radial } \\
\text { wrist extensors, pectoralis } \\
\text { major }\end{array}$ & $\begin{array}{l}\text { Shoulder lateral rotation, } \\
\text { abduction/elbow flexion, } \\
(+/-) \text { wrist extension }\end{array}$ & $\begin{array}{l}\text { Thumb and index } \\
\text { finger }\end{array}$ \\
\hline C5/6/7 & $\begin{array}{l}\text { As above and triceps, ECRL, } \\
\text { FCR, EDC, EPL, EPB, APL }\end{array}$ & $\begin{array}{l}\text { As above, plus elbow, } \\
\text { wrist, finger, and thumb } \\
\text { extensors }\end{array}$ & $\begin{array}{l}\text { As above, plus } \\
\text { middle finger }\end{array}$ \\
\hline $\mathrm{C}(7) / \mathrm{C} 8 / \mathrm{T} 1$ & $\begin{array}{l}\text { (EDC, EPL), FDS, FDP, FPL, } \\
\text { lumbricals, interossei, thenar, } \\
\text { hypothenar }\end{array}$ & $\begin{array}{l}\text { (Finger extension), } \\
\text { finger+thumb flexion, } \\
\text { median/ulnar intrinsics }\end{array}$ & $\begin{array}{l}\text { (Middle finger), } \\
\text { little/ring fingers }\end{array}$ \\
\hline C5 to T1 & All of above & All of above & All of above \\
\hline
\end{tabular}

Fensor carpi radialis longus; FCR: Flexor carpi radialis; EDC: Extensor digitorum communis; EPL: Extensor pollicis longus; EPB: Extensor pollicis brevis; APL: Abductor pollicis longus; FDS: Flexor digitorum superficialis; FDP: Flexor digitorum profundus; FPL: Flexor pollicis longus.

In this case, from physical examination, we found positive winging scapula in the left shoulder, $5 / 1$ shoulder abduction, and C5 high hypoesthesia. The patient can extend his elbows, wrists, and all five fingers, so the possibility of $\mathrm{C} 7$ lesions can be temporarily removed. Based on these findings and the suitability of findings in brachial plexus lesions, the patient was suspected of having lesions as high as C5/C6 left.

Further investigation needs to be done to confirm clinical findings. X-ray imaging of the cervical vertebrae, including the shoulders, extremities, and chest, must be worked out to exclude the possibility of fractures of the spine, ribs, clavicle, scapula, or extremity bones around the gunshot wound. Discovery of fractures in ribs I and II indicates the possibility of injury to the brachial plexus [5]

Myelography is a simple, economical diagnostic method, and available in most hospitals but has disadvantages in the form of radiation exposure and the adverse effects of contrast material. This examination is able to show avulsion of nerve roots with a water-soluble contrast agent. Its accuracy reaches $75 \%$ in lesions as high as C8 and $\mathrm{T} 1$ and decreases in nerve root lesions as high as C5-C6. This may be due to the narrow subarachnoid space at that level. At present, computed tomography (CT) myelography is the gold standard as a diagnostic method of nerve avulsion, which able to evaluate ventral and dorsal nerve roots separately and intradural nerve defects 
are detected. The big advantage of CT myelography compared to myelogram is its ability to detect partial avulsion at levels C5 and C6, with an overall accuracy of $75 \%$ [5].

Magnetic resonance imaging (MRI) has shown less accurate imaging compared with CT myelography. The relative advantages of MRI versus CT myelography are non-invasive and the brachial plexus image quality is much better. MRI is also useful for visualizing postganglionic lesions and for differentiating from benign and malignant tumor lesions. Ultrasonography (USG) can be an option in evaluating the post-ganglionic brachial plexus. The advantages of ultrasound include the resolution of high soft tissue and the ability to identify each part of the plexus as it passes through the oblique plane at the base of the neck. However, the reliability of this technique in post-ganglionic injury cases still requires further explanation [5]

EMG is an electrodiagnostic modality to evaluate the level of electrical activity produced by skeletal muscle. EMG is very helpful for confirming the diagnosis, localizing the lesion level, estimating the severity of axon loss, and completeness of the lesion. It also helps distinguish pre-ganglionic lesions from post-ganglionic lesions. The potential for sensory nerve action is particularly important in localizing pre- or postganglionic lesions. Serial EMG with repeated physical examinations is needed to identify the progression of recovery. The presence of an active motor unit, the appearance of the initial potential, and a decrease in the amount of fibrillation potential indicate a good recovery and prognosis [5].

In our case, with the suspicion of C5-C6 high left brachial plexus lesions, conventional AP chest and left AP shoulder images were taken 3 months after the gunshot wound. The examination was carried out at the Bhayangkara Hospital in Palu. In the AP chest radiograph, complete fractures of the middle third of the clavicle appear and other structures are within normal limits. The left AP shoulder photo confirms the fracture of the middle third of the left clavicle os. Cervical photos in the case were not treated to see the awareness of patients who had compost mentis, normal neck mobility, and no injury to the colli region. Exploration and debridement measures were taken immediately to look for possible neurovascular lesions. The patient is then treated with internal reduction and fixation using plate screw. The patient complained that it was still difficult to move his upper limb after 2 months after the procedure. The patient was referred to Sanglah General Hospital and the patient had been given an EMG examination, and the conclusion was that there were lesions in the left posterior cord and the left brachial plexus nerve.

Panell et al. found $63 \%$ of cases with nerve dysfunction after GSWs, and neurological deficits were significantly associated with nerve contusion or intraoperative lacerations. About $75 \%$ of all patients with nerve palsy are associated with nerve lacerations during exploratory surgery. However, other studies found that the act of exploration and repair as early as possible increased the risk of better functional recovery compared to merely observing. Early exploration allows the clinician to identify nerve lacerations, the possibility of reconstruction, and helps determine the therapy and monitoring that is needed in the future. Panell et al. recommend early exploration in patients after GSWs to the upper extremities, especially in patients who will undergo surgical treatment for other indications, such as irrigation and debridement, fracture fixation, or vascular reconstruction [1]. Based on these studies, the likelihood of previous surgical exploration actions contributes to the emergence of brachial plexus lesions that patients experience. However, we should not deny the importance of early exploration in cases of GSWs to achieve optimal function recovery in patients.

Careful physical examination is mandatory to assess the motor and sensory functions of each muscle group that receives innervation from the brachial plexus to determine which therapy is given. In addition, understanding the mechanism, onset, type (pre- or postganglionic), the priorities of treatment, and concomitant injuries should be considered in decision-making process. Conservative therapy has a place when EMG results show non-degenerative lesions. Conservative therapy consists of use of assistive devices such as slings or splints to minimize the uncontrolled movement of paralytic limbs. Extensive physical therapy, passive range of movements (PROMS), electrical stimulation, and massage are also included in conservative therapy. Pain management is also a part of conservative therapy which is very important to minimize discomfort and maximize other therapeutic potential [5].

Surgical therapy is generally indicated when spontaneous recovery is not achieved after 6 months after injury. Even so, until now, the best time for reconstructive surgery to be done is still a debate. Surgery that is too early can interfere with the spontaneous reinnervation process, but late surgical procedures after 6-18 months of denervation can lead to motor end-plate formation failure which ends in failure to reinnate. In general, optimal time is set between 3 and 6 months after trauma. The mechanism of trauma also determines the timing of surgical intervention. Surgery needs to be done immediately if there is vascular trauma. If there is complete avulsion of nerve fibers $\mathrm{C} 5$ and $\mathrm{T} 1$, exploration must be done within 1-2 weeks after trauma. When suspicion of avulsion of nerve fibers is very high, exploratory action must be carried out within 3-6 weeks post-trauma. Moreover, if the function or physical examination or EMG does not show improvement, delayed exploration must be done in the $12^{\text {th }}$ week post-trauma. Gunshot wound is also an indication of immediate exploration [6]. Surgical procedure that can be done in cases of brachial plexus trauma, namely: 


\section{Nerve grafting}

Nerve grafting is a procedure that is often done to connect broken nerve fibers in post-ganglionic lesions. This action can trigger regeneration of axons according to the old pathway to innervate some muscles. However, this action cannot be performed in pre-ganglionic lesions. The most commonly used nerve donor is the sural nerve which can reach $30 \mathrm{~cm}$ in length. The most common used nerve donor is the sural nerve which can reach $30 \mathrm{~cm}$ in length. The frequent procedure perform such as; C5 fibers to the supraclavicular nerve or axillary nerve, C6 fibers to the musculocutaneous nerve, and $\mathrm{C} 7$ fibers to the tricep nerve or radial nerve [6]. Chuang et al. compared grafts of $<10 \mathrm{~cm}$ in size with graft sizes of more than $10 \mathrm{~cm}$ and concluded that the length of the graft was also an important factor determining the success of the nerve grafting procedure [7]. Shorter sized grafts have a higher success rate of reinnervation. This also indicates shorter brachial plexus lesions tend to have a better prognosis of reinnervation after the action [8].

Surgical techniques are an important factor in determining the success of surgery. Weak coaptation and tension at the site of nerve union will increase the scar tissue formation, fibrosis, and granulomatosis, which may worsen the redevelopment of growing axons [5].

\section{Neurotization (nerve transfer)}

This procedure seems to be applicable to preganglionic lesions. The purpose of nerve transfers is not to form reinnervation such as nerve grafting, but donor nerves are connected to the butts of nerve fibers that break or are sacrificed to achieve more important nerve functions (recipient nerves). Nerve transfer can return the axonal input to the denervation target. One of the advantages of this procedure is the closeness

Table 2: The donor nerve and recipient of the nerve transfer procedure [5]

\begin{tabular}{ll}
\hline Donor nerves & Recipient nerves \\
\hline Spinal accessory nerve & Suprascapular nerve \\
& Musculocutaneous nerve \\
Phrenic nerve & Axillary nerve \\
& Suprascapular nerve \\
& Musculocutaneous nerve \\
Intercostal nerve & Axillary nerve \\
& Upper trunk \\
& Musculocutaneous nerve \\
& Long thoracic nerve \\
& Radial nerve \\
& Median nerve \\
Contralateral C7 nerve & Axillary nerve \\
& Medial pectoral nerve \\
Cervical plexus & Ulnar nerve \\
& Median nerve \\
& Musculocutaneous nerve \\
& Lateral cord \\
Hypoglossal nerve & Axillary nerve \\
& Median nerve \\
& Musculocutaneous nerve \\
& Suprascapular nerve \\
& Radial nerve \\
& Musculocutaneous nerve \\
& Lateral cord \\
\hline
\end{tabular}

of the donor's anatomy and the nerve of the recipient, and reduced operating time and does not require grafts or grafts. In general, biceps muscle function and shoulder function are considered to have priority in nerve reconstruction. Intercostal-musculocutaneous nerve transfer is usually used to regain elbow flexion, and transfer of the accessory-suprascapular nerve is used to obtain functional recovery from the shoulder (Table 2) [6].

We report a case of the left upper extremity weakness due to brachial plexus trauma after GSWs that have been confirmed through EMG examination. The patient has been performed a nerve transfer procedure with an accessory nerve donor to the supraclavicular nerve to restore the patient's left shoulder functionally. Results from reconstructive surgery have been widely reported, in which the most cases get their motor function after surgical reconstruction procedure [8].

\section{Conclusions}

Brachial plexus lesion either due to trauma or non-trauma mechanism has a great impact on the patient's quality of life. However, advances in surgical techniques and further understanding of nerve physiology have brought clinicians and patients to better outcomes. The current trend of treatment strategies for brachial plexus trauma is a good surgical reconstruction with the nerve transfer procedure.

\section{References}

1. Pannell WC, Heckmann N, Alluri RK, Sivasundaram L, Stevanovic M, Ghiassi A. Predictors of nerve injury after gunshot wounds to the upper extremity. Hand. 2017;12(5):501-6. https:// doi.org/10.1177/1558944716675294

PMid:28832207

2. Centers for Disease Control and Prevention. NonFatal Data WISQARS Injury Center. Atlanta, Georgia: Centers for Disease Control and Prevention. Available from: https://www.cdc.gov/ injury/wisqars/nonfatal.html. [Last accessed on 2021 May 02]. https://doi.org/10.1037/e309102004-001

3. Sathiyakumar V, Thakore RV, Stinner DJ, Obremskey WT, Ficke JR, Sethi MK. Gunshot-induced fractures of the extremities: A review of antibiotic and debridement practices. Curr Rev Musculoskelet Med. 2015;8(3):276-89. https://doi. org/10.1007/s12178-015-9284-9

PMid:26047781

4. Rasulić L, Simić V, Savić A, Lepić M, Kovačević V, Puzović V, et al. Management of brachial plexus missile injuries. Acta Clin Croat. 2018;57(3):487-96. https://doi.org/10.20471/acc.2018.57.03.12 PMid:31168182

5. Park HR, Lee GS, Kim IS, Chang JC. Brachial plexus injury in adults. Nerve 2017;3:1-11. 
6. Sakellariou VI, Badilas NK, Stavropoulos NA, Mazis G, Kotoulas HK, Kyriakopoulos S, et al. Treatment options for brachial plexus injuries. ISRN Orthop. 2014;2014:314137. https://doi.org/10.1155/2014/314137

PMid:24967125

7. Chuang DC, Epstein MD, Yeh MC, Wei FC. Functional restoration of elbow flexion in brachial plexus injuries: Results in 167 patients (excluding obstetric brachial plexus injury). J Hand Surg Am. 1993;18(2):285-91. https://doi. org/10.1016/0363-5023(93)90363-8

\section{PMid:8463596}

8. Secer HI, Solmaz I, Anik I, Izci Y, Duz B, Daneyemez MK, et al. Surgical outcomes of the brachial plexus lesions caused by gunshot wounds in adults. J Brachial Plex Peripher Nerve Inj. 2009;4:11. https://doi.org/10.1186/1749-7221-4-11

PMid:19627573 